\title{
TWO-STREAM INSTABILITY ANALYSIS FOR PROPAGATING CHARGED PARTICLE BEAMS WITH A VELOCITY TILT*
}

\author{
D. V. Rose ${ }^{\dagger}$, T. C. Genoni, D. R. Welch, Voss Scientific, LLC, Albuquerque, NM 87108, USA \\ R. C. Davidson, E. A. Startsev, Princeton Plasma Physics Laboratory, Princeton, NJ 08543, USA
}

\begin{abstract}
The linear growth and saturation of the two-stream instability for a charged particle beam that is longitudinally compressing as it propagates through a background plasma (due to an applied velocity tilt) has recently been examined. Detailed, 1D particle-in-cell simulations are carried out to examine the growth and saturation of a wavepacket produced by a small amplitude density perturbation in the background plasma. The resultant electrostatic wave spectra that evolves due to the coupling of the primary (resonant) unstable wave packet with the plasma is examined.
\end{abstract}

\section{INTRODUCTION}

Longitudinal drift compression of ion beams is being studied as a means to achieve short-duration, high-energydensity pulses for warm-dense-matter [1] and heavy ion inertial fusion energy [2] applications. Longitudinal compression is driven by a velocity profile or tilt on a charged particle beam that is applied either at the source as part of the beam generation (as in some high power diodes) or by passing the beam through a programmed accelerating structure.

Recent experiments at Lawrence Berkeley National Laboratory (LBNL) have successfully demonstrated controlled longitudinal drift compression of heavy ion beams [3]. A velocity tilt was applied to an unneutralized, drifting ion beam using an induction core, and then the beam entered a plasma-filled drift region where longitudinal compression factors, $I_{b F} / I_{b 0}$, over 60 were achieved, where $I_{b 0}$ and $I_{b F}$ are the initial and peak compressed beam currents, respectively. At the end of this drift region, radial compression of the beam will be attempted by passing the longitudinally compressing beam through a plasma-filled solenoid.

Theoretical modeling supporting both the design and analysis of these experiments has been carried out using analytic methods [4] and numerical simulations [5]. As this modeling is extended to parameter regimes beyond current experimental values to future high-energy-density applications, careful consideration of the growth and saturation of a number of potential beam-plasma instabilities must be considered (see, for example, Ref. [6]). In particular, recent work has examined the growth of the two-stream instability for longitudinally compressing charged particle beams propagating in background plasmas [7].

\footnotetext{
${ }^{*}$ Research supported by U. S. DOE through Princeton Plasma Physics Laboratory for the Heavy Ion Fusion Science Virtual National Laboratory

$\dagger$ david.rose@vosssci.com
}

Here, we seek estimates for the electrostatic wave energy spectrum resulting from the growth and saturation of the two-stream instability in a background plasma using particle-in-cell (PIC) simulations. The PIC model can also be used to examine instability saturation mechanisms and other nonlinear effects, which are difficult to assess from a purely analytic approach. The 1D PIC simulation model is described below and the asymptotic phase of the beam-plasma system is analyzed. The basic features of the electrostatic wave spectrum from the simulations is analyzed and applied to theoretical estimates of the longitudinal emittance growth of an ion beam propagating in the resultant weakly or moderately turbulent plasma.

\section{D SIMULATION MODEL}

The PIC code LSP [8] is used in 1D to examine the evolution of electrostatic turbulence initially driven the twostream instability for charged particle beams propagating in a background plasma. The simulations are electrostatic and use a cloud-in-cell model to help minimize electrostatic fluctuations on the grid. A previous analysis compared 1D PIC simulations results to wave energy densities from a dynamic wave-population model, confirming asymptoticphase wave-coupling mechanisms, including electrostatic turbulence levels [9].

A perturbation of the plasma electron density at $t=0$ and $x=50 \mathrm{~cm}$ of the form

$$
n_{p e}= \begin{cases}n^{\dagger} \exp (-a|x|), & \text { for } x>0, \\ -n^{\dagger} \exp (-a|x|), & \text { for } x<0,\end{cases}
$$

is used, where $n^{\dagger}$ is the density perturbation amplitude and the coefficient $a$ determines the characteristic width of the perturbation. This provides an impulse-like electric field that excites a growing wavepacket.

The simulations use four separate charged-particle species to represent the (initially) charge and currentneutral beam-plasma system. Electrons and ions with equal velocity and number density (with $m_{b i}=10^{9} m_{e}$ or $27 m_{p}$, where $m_{b i}, m_{e}$, and $m_{p}$ are the beam ion, electron, and proton masses) are initially propagating in a charge-neutral, cold-background electron-ion plasma $\left(m_{p i}=10^{9} m_{e}\right.$ or $39 m_{p}$, where $m_{p i}$ is the plasma ion mass). The $10^{9} m_{e}$ ion masses are used in some of the simulations to selectively eliminate ion-electron and ion-ion modes from the analysis.

The simulations are typically initialized with 100 particles per cell (combined beam and plasma particles), $\Delta t=$ $3 \times 10^{-4} \mathrm{~ns}$, and $\Delta x=0.01 \mathrm{~cm}$ or $0.001 \mathrm{~cm}$, where $\Delta t$ 


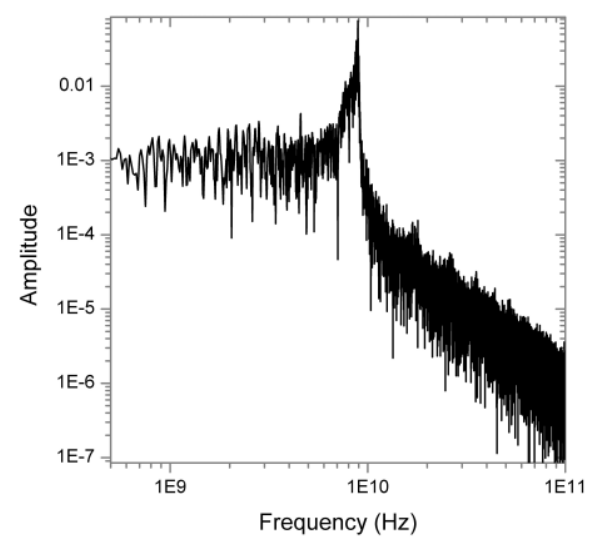

Figure 1: Fourier transform of the electric field at $x=$ $75 \mathrm{~cm}, 25 \mathrm{~cm}$ downstream of the initial perturbation position. Here $m_{b i}=m_{p i}=10^{9} m_{e}$.

and $\Delta x$ are the time step and cell size. Nominal two-stream wavelengths for our parameters are $\lambda=2 \pi V_{b 0} / \omega_{p e} \simeq$ $0.33 \mathrm{~cm}$, which are well-resolved by $\Delta x$ as are plasma oscillations $\omega_{p e} \Delta t \simeq 0.017<1 / 2$. The simulation box is $100 \mathrm{~cm}$ in length and the perturbation described in Eq. (1) is initialized at center of the simulation. The beam speed for this sample simulation is $V_{b 0}=0.1 c$, where $c$ is the speed of light. Periodic boundaries are used to eliminate space charge waves that are driven by typical sheath formation at conducting boundaries. Consideration of these and other effects guided the selection of the parameters, balancing growth and saturation of the initial wavepacket from the initial programmed perturbation against non-ideal effects.

\section{RESULTS}

Results of several PIC simulations are summarized here. For all cases the plasma density is $10^{12} \mathrm{~cm}^{-3}$, the beam density is $10^{8} \mathrm{~cm}^{-3}, a=5 \mathrm{~cm}^{-1}$, and $n^{\dagger}=10^{6} \mathrm{~cm}^{-3}$. The simulations follow the linear and nonlinear growth of the two-stream instability over many exponential growth times until nonlinear effects such as particle-trapping lead to saturation of the instability growth. At this point, the combined background plasma and beams constitute a somewhat turbulent plasma containing a spectrum of longitudinal electrostatic waves. The frequency spectrum of these waves can be observed in Fig. 1, which plots the Fourier spectrum of the electric field $25 \mathrm{~cm}$ downstream of the initial perturbation. The peak in the spectrum occurs at the electron plasma frequency, $f_{e}$. The long plateau at frequencies below $f_{e}$ here represents a portion of the spectrum that has evolved in the system.

A more complete picture of the evolution of the electrostatic turbulence is given in Fig. 2, which plots the magnitude of the electric field in both time and space. The perturbation is initialized at $(x, t)=(50 \mathrm{~cm}, 0)$ and the line ex-

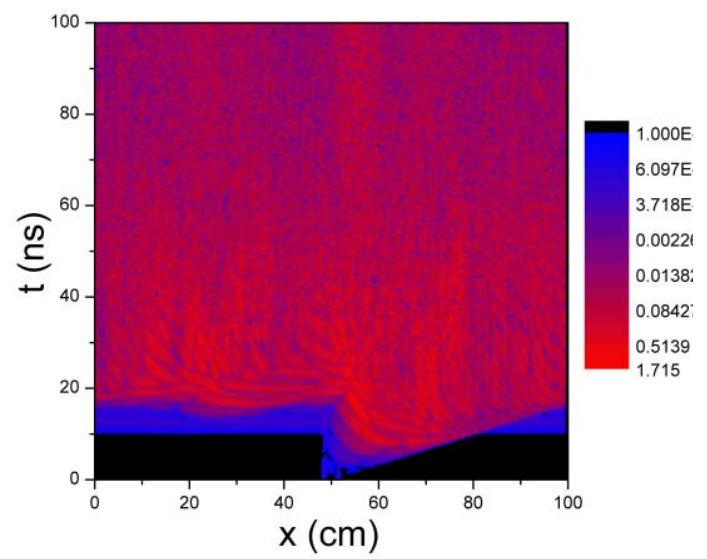

Figure 2: Contour plot of the magnitude of the electric field in $(x, t)$. Here $m_{b i}=m_{p i}=10^{9} m_{e}$.

tending forward in both $x$ and $t$ from this point is the initial unstable wave-packet trajectory. Immediately above this line, the evolution of the initial growth of the two-stream instability can be observed until approximately $t=15 \mathrm{~ns}$, when the nonlinear effects drive waves that couple to the entire spatial extent of the system. For times greater than $\sim 40 \mathrm{~ns}$, the system enters a quasi-equilibrium phase where the main features of the spectrum discussed in reference to Fig. 1 are evident.

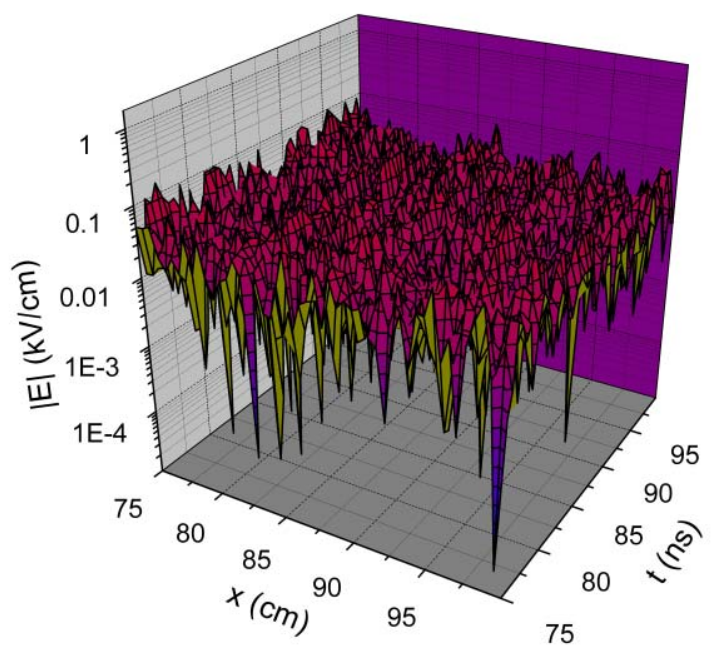

Figure 3: Three-dimensional view of the electric field from Fig. 2.

A more detailed view of this quasi-equilibrium phase is given in Fig. 3, which is a 3D plot of the electric field amplitude in the range $75 \leq x \leq 100 \mathrm{~cm}$ and $75<t<$ $100 \mathrm{~ns}$. A variety of wavelengths and frequencies can be observed that characterize the electrostatic turbulent spectrum. 


\section{LONGITUDINAL EMITTANCE GROWTH}

We seek estimates of the longitudinal emittance growth rate for a cold charged particle stream passing through a turbulent plasma. A number of authors have developed models for the velocity diffusion of charged particles in the presence of electrostatic and electromagnetic turbulence. These include Fokker-Plank models where detailed calculations of the relevant diffusion coefficients are developed [10], as well as stochastic descriptions [11]. We note that models of stochastic electrostatic fields are likely to be applicable to the PIC simulations presented above.

As a first look, we consider the following simple description for the late-time electric field that evolves in the PIC simulations,

$$
E(x, t)=E_{0} E_{x}(x) E_{t}(t)
$$

where

$$
\begin{aligned}
& E_{x}(x)=\sum_{i=1}^{3} A_{i} \cos \left(2 \pi x / L_{i}\right), \\
& E_{t}(t)=\sum_{j=1}^{2} B_{j} \sin \left(2 \pi t / T_{j}\right) .
\end{aligned}
$$

This simple description is inspired by the electromagnetic turbulence models developed in [12] and the parameters are adapted from analysis of the PIC simulation results. A plot of the electric field map is given in Fig. 4 for the following parameters: $E_{0}=0.5 \mathrm{kV} / \mathrm{cm}, A_{i}=(0.1,0.2,0.7), L_{i}=$ $(2.5,0.5,0.33) \mathrm{cm}, B_{j}=(0.8,0.2), T_{j}=(0.25,1.25) \mathrm{ns}$.

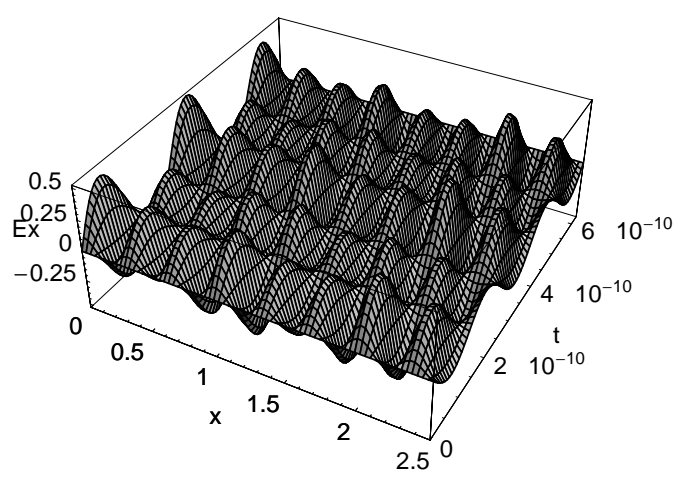

Figure 4: 3D view of $E(x, t)$ given by Eq. (2).

Injection of a cold $255 \mathrm{kV}, \mathrm{K}^{+1}$ ion stream into a periodic transport region results in a small spread in ion velocities after $1.1 \mathrm{~m}$ of transport, as shown in Fig. 5. In this simple model, the energy spread increases very slowly, scaling approximately as the square root of the propagation distance.

The simple model examined here, in conjunction with the PIC simulations discussed above, confirms the absence of any measurable longitudinal emittance growth due to the propagation of an ion beam through a pre-formed plasma in the LBNL experiments [13]. These models will be applied

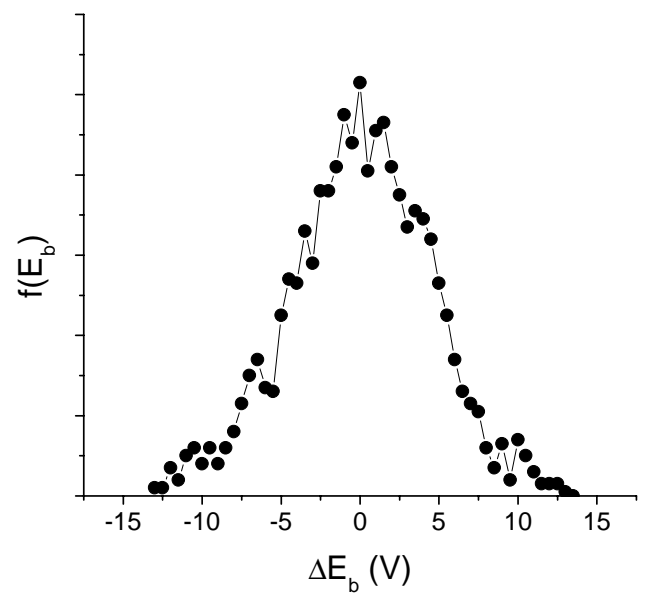

Figure 5: Sample calculation of ion beam longitudinal emittance growth. For this example, $255 \mathrm{kV}, \mathrm{K}^{+1}$ test ions were transported through the longitudinal electric field described by Eq. (2).

to other parameters to develop a scaling for longitudinal emittance growth in future laboratory experiments as well as astrophysical problems including cosmic ray propagation.

\section{REFERENCES}

[1] N. A. Tahir, et al., Contrib. Plasma Phys. 43, 373 (2003); D. H. H. Hoffmann, et al., Phys. Scr. T123, 1 (2006).

[2] B. G. Logan, et al., Nucl. Fusion 45, 131 (2005).

[3] R. K. Roy, et al., Phys. Rev. Lett. 95, 234801 (2005).

[4] E. A. Startsev and R. C. Davidson, New J. Phys. 6, 141 (2004).

[5] H. Qin, et al., Phys. Rev. ST-AB 7, 104201 (2004); D. R. Welch, et al., Nucl. Instrum. Meth. Phys. Res. Sect. A 544, 236 (2005); C. Thoma, et al., Phys. Plasmas 12, 043102 (2005); A. B. Sefkow and R. C. Davidson, Phys. Rev. STAB 9, 090101 (2006); D. R. Welch, et al., Nucl. Instrum. Meth. Phys. Res. Sect. A 577, 231 (2007); D. R. Welch, et $a l$. , these proceedings.

[6] R. C. Davidson, et al. Phys. Rev. ST-AB 7, 114801 (2004); R. C. Davidson, et al. Nucl. Instrum. Meth. Phys. Res. A, 577, 70 (2007).

[7] E. A. Startsev and R. C. Davidson, Phys. Plasmas 13, 062108 (2006); D. V. Rose, et al., Phys. Rev. ST-AB 10, 034203 (2007).

[8] LsP is a software product of ATK-Mission Research, Albuquerque, NM 87110.

[9] D. V. Rose, et al., Phys. Plasmas 9, 1000 (2002).

[10] J. M. Pryadko and V. Petrosian, Ap. J. 482, 774 (1997); J. Struckmeier, Phys. Rev. ST-AB 3, 034202 (2000).

[11] R. W. Fredricks, J. Plasmas Phys. 1, 241 (1967); G. Knorr, Plasma Phys. 11, 917 (1969).

[12] R. G. Kleva, Phys. Fluids B 3, 3312 (1991).

[13] P. A. Seidl, et al., Nucl. Instrum. Meth. Phys. Res. A, 577, 215 (2007).

D03 High Intensity - Incoherent Instabilities, Space Charge, Halos, Cooling 